\title{
Characterisation of the ex vivo virulence of Leishmania infantum isolates from Phlebotomus perniciosus from an outbreak of human leishmaniosis in Madrid, Spain
}

Gustavo Domínguez-Bernal', Maribel Jiménez², Ricardo Molina², Lara Ordóñez-Gutiérrez ${ }^{3}$, Abel Martínez-Rodrigo ${ }^{1}$, Alicia Mas ${ }^{1}$, Maria Teresa Cutuli ${ }^{1}$ and Javier Carrión ${ }^{1 *}$

\begin{abstract}
Background: Since mid 2009, an outbreak of human leishmaniosis in Madrid, Spain, has involved more than 560 clinical cases. Many of the cases occurred in people who live in areas around a newly constructed green park (BosqueSur). This periurban park provides a suitable habitat for sand flies (the vectors of Leishmania infantum). Indeed, studies of blood meals from sand flies captured in the area showed a strong association between the insect vector, hares or rabbits, and humans in the area. Interestingly, up to $70 \%$ of cases have been found in immunocompetent patients (aged between 46-60 years). This study was designed to evaluate the ex vivo virulence of the L. infantum isolates from Phlebotomus perniciosus captured in this area of Madrid.

Methods: Murine macrophages and dendritic cells were infected ex vivo with L. infantum strain BCN150, isolate BOS1FL1, or isolate POL2FL7. At different times after infection, the infection indices, cytokine production (IL-12p40 and IL-10), NO release and arginase activities were evaluated.

Results: Using an ex vivo model of infection in murine bone marrow-derived cells, we found that infection with isolates BOS1FL1 and POL2FL7 undermined host immune defence mechanisms in multiple ways. The main factors identified were changes in both the balance of iNOS versus arginase activities and the equilibrium between the production of IL-12 and IL-10. Infection with isolates BOS1FL1 and POL2FL7 also resulted in higher infection rates compared to the BCN150 strain. Infection index values at $24 \mathrm{~h}$ were as follows: BCN150-infected cells, 110 for infected $M \varnothing$ and 115 for infected DC; BOS1FL1-infected cells, 300 for infected M $\varnothing$ and 247 for infected DC; and POL2FL7-infected cells, 275 for infected M $\varnothing$ and 292 for infected DC.

Conclusions: Our data indicate that $L$. infantum isolates captured from this endemic area exhibited high virulence in terms of infection index, cytokine production and enzymatic activities involved in the pathogenesis of visceral leishmaniosis. Altogether, these data provide a starting point for the study of the virulence behaviour of parasites (BOS1FL1 and POL2FL7) isolated from P. perniciosus during the outbreak of human leishmaniosis in Madrid, Spain, and their involvement in infecting immunocompetent hosts.
\end{abstract}

Keywords: Leishmania infantum, Phlebotomus perniciosus, Leishmaniosis, Outbreak, Virulence, BosqueSur, Madrid, Spain

\footnotetext{
* Correspondence: fjcarrion@vet.ucm.es

${ }^{1}$ Department of Animal Health, Faculty of Veterinary Science, Complutense University of Madrid, 28040 Madrid, Spain

Full list of author information is available at the end of the article
} 


\section{Background}

Leishmania infantum is the etiologic agent of zoonotic visceral leishmaniosis (VL) in humans and other mammals (dogs are the main domestic reservoir hosts) in all countries of the Mediterranean basin [1,2]. VL is a potentially fatal disease in dogs. In several areas of Spain where canine leishmaniosis is highly endemic, seroprevalence of up to $34 \%$ has been found, and is considered to be a major problem $[3,4]$. Other animals including rabbits, hares and cats have also been considered as possible alternative reservoirs of VL [5-7]. Parasites are transmitted from vertebrate animals to humans by the bite of infected female phlebotomine sandflies. No effective vaccine exists against any form of human leishmaniosis. The spectrum and efficacy of current antileishmanial drugs are also limited [8]. All these aspects have recently been reviewed in detail elsewhere [9].

Active VL, mainly diagnosed in young children and in immunocompromised adults, results from uncontrolled multiplication of the parasite in phagocytes of the reticuloendothelial system, and infection is lethal if left untreated. Although the incidence of active disease in humans is relatively low (a range of approximately 5-200 VL cases/ year reported by each country), cases are increasing and spreading $[10,11]$.

The environment is undergoing rapid changes because of human actions [12] that also contribute to the changing landscape of leishmaniosis. The latter includes increasing risk factors and new scenarios associated with the zoonotic VL $[1,11]$. In Spain, a district of abandoned farmland and tailings areas surrounded by a large urban population in the southwest of Madrid was chosen as a site for a newly constructed periurban green park (BosqueSur). This area has been associated with an outbreak of human leishmaniosis, and more than 560 human cases have been detected from July 2009 to date; notably, $70 \%$ of the cases have been found in immunocompetent patients aged between $46-60$ years $[13,14]$.

Current studies suggest that this outbreak was due to human-induced environmental changes (land cover and land use) that created regional combinations of eco-epidemiological conditions; these conditions may influence patterns of sand fly vector ( $P$. perniciosus) distribution and may result in infections of unusual mammalian hosts of L. infantum [14-17]. In this context, xenodiagnostic studies performed with wild lagomorphs (hares and rabbits) captured in the green park demonstrate that these animals play some role as unusual reservoirs that transmit L. infantum parasites to $P$. perniciosus. Moreover, studies of blood meal preferences of $P$. perniciosus caught in the area revealed that the insect vector prefers to feed on rabbits and hares, but will occasionally feed on other hosts, such as dogs or humans $[14,18,19]$. Altogether, published data support the idea that the peridomestic and sylvatic transmission cycles overlap in the area, and that infected dogs are not essential to maintaining the transmission cycle of L. infantum.

Since this outbreak started, lagomorphs have attracted the interest of researchers in Spain. Chicharro et al. [20] suggested that the outbreak was not caused by a 'new' emerging genotype. Molecular typing studies show that the isolates involved in the outbreak belonged to the ITS LOMBARDI subtype of L. infantum, as did those isolated in different parts of Madrid since at least 1992.

However, the question regarding how parasites have been able to infect a high number of immunocompetent patients during the current outbreak remains important and unanswered. The aim of the present study was to evaluate the ex vivo virulence of the $L$. infantum isolates recovered from $P$. perniciosus that were captured in the area of human leishmaniosis in Madrid, in comparison with that of another well-characterized strain.

\section{Methods}

\section{Mice and parasites}

Six-week-old female BALB/c mice were purchased from Harlan Interfauna Ibérica (Barcelona, Spain). The animal research described in this manuscript complied with Spanish (Ley 6/2013) and European Union legislation (2010/63/ UE). The protocols used were approved by the Animal Care Committee of Complutense University of Madrid.

L. infantum parasites were used in this study. Two isolates named IPER/ES/2012/BOS1FL1 (BOS1FL1) and IPER/ES/2012/POL2FL7 (POL2FL7) were isolated from $P$. perniciosus captured in the focus of Madrid using CDC light traps. BCN150 is a well characterized strain (M/CAN/ES/96/BCN150 zymodeme MON-1) that was isolated from a dog with active VL [21]. This strain has traditionally been used in our laboratory's experiments related to VL [22-24] and by others in canine leishmaniosis studies $[21,25,26]$. All L. infantum parasites were previously passed through golden hamsters (Mesocricetus auratus). Two months after infection, infected spleen samples were cultivated in NNN medium at $26-27^{\circ} \mathrm{C}$ for 4-7 days, until promastigotes expanded. These were then used for ex vivo infection experiments in this study.

\section{Differentiation of bone marrow-derived cells}

Macrophages (BMMø) and dendritic cells (BMDC) were differentiated in vitro from bone marrow stem cell progenitors. Briefly, BALB/c mice were euthanised, and cell suspensions were obtained by flushing the femurs and tibias with Hank's Balanced Salt Solution (HBSS). Cells were collected by centrifugation and cultured in DMEM containing $2 \mathrm{mM}$ L-glutamine, $0.1 \mathrm{mM}$ nonessential amino acids, $10 \mathrm{mM}$ HEPES, antibiotics, and 10\% heatinactivated foetal bovine serum (FCS). BMMø were cultured in non-tissue culture treated Petri dishes for 8 
days in the presence of M-CSF (Peprotech) at a concentration of $50 \mathrm{ng} / \mathrm{ml}$. At days 3 and 6 , half of the supernatants were discarded and replaced with $5 \mathrm{ml}$ of fresh medium containing M-CSF $(50 \mathrm{ng} / \mathrm{ml})$. BMDC were cultured for 10 days in $25 \mathrm{ml}$ flasks in the presence of GMCSF (20 ng/ml, Peprotech). This supplemented DC medium was added at days 0,3 and 6 . After their respective periods of differentiation, BMMø and BMDC were obtained as previously described $[27,28]$ and displayed a phenotype highly enriched in $\mathrm{F} 4 / 80^{+}$or $\mathrm{CD} 11 \mathrm{c}^{+}$ cells $(\sim 95 \%)$, respectively.

\section{Infection index of bone marrow-derived cells}

$\mathrm{BMM} \varnothing$ and BMDC were cultured overnight in the presence or absence of IFN- $\gamma(500 \mathrm{ng} / \mathrm{ml})$ into LabTek culture chamber slides (Thermo Scientific) using $5 \times 10^{4}$ cells per chamber. On the following day, parasites were added to cells at a ratio of 10:1 parasites: BMMø or BMDC. After 4 $\mathrm{h}$ of incubation at $37^{\circ} \mathrm{C}$, a time point that reflects initial infection, extracellular parasites were removed by washing, and cells were incubated in fresh medium for $24 \mathrm{~h}, 48 \mathrm{~h}$ and $72 \mathrm{~h}$. After Giemsa staining, cells were mounted with Coverquick, and 400 cells were counted in duplicate in a microscope Olympus BX41. The percentage of infected cells and the mean of the number of amastigotes per infected cell were evaluated. The infection index was calculated by multiplication of both parameters to account for the overall parasite load, as previously described [29].

\section{Cytokine production, nitric oxide (NO) release and arginase activity}

BMMø and BMDC $\left(1 \times 10^{6}\right.$ cells $\left./ \mathrm{ml}\right)$ were cultured overnight into 24 well non-tissue culture treated plates. Thereafter, parasites were added (at a ratio of 10:1, as above) to cells and extracellular promastigotes were removed by washing after $4 \mathrm{~h}$. Subsequently, cells were stimulated or not with LPS $(1 \mu \mathrm{g} / \mathrm{ml})$. In some cases, culture supernatants were collected after $24 \mathrm{~h}$ for cytokine quantification (IL-12p40 and IL-10, BD Pharmigen) by ELISA according to the manufacturer's instructions. At $96 \mathrm{~h}$, supernatants from the other wells were collected and nitric oxide NO release was measured as nitrite concentration using Griess reagent. Then, BMMø and BMDC were then harvested and used to determine of arginase activity as previously described [30].

\section{Statistical analysis}

Statistical analyses were performed using SigmaPlot version 11.0 (Systat Software, Inc). Significant differences between different strains were determined and are designated with asterisks as follows: ${ }^{*} P<0.05,{ }^{* *} P<0.01$, ${ }^{* * *} P<0.001$.

\section{Results and discussion}

In this study, we defined virulence in terms of measuring the abilities of a well-characterised L. infantum strain and two isolates from $P$. perniciosus to induce factors contributing to disease under controlled conditions. In this ex vivo context, we quantified virulence, using assessment of both infection index and the quality of immune responses due to infection, as shown below.

\section{Evaluation of infection index and related markers}

We infected murine BMMø and BMDC and followed the progression of infection for $72 \mathrm{~h}$. At the initial time point studied $(4 \mathrm{~h})$, more than $80 \%$ of the cells were infected, and there were no significant differences between parasite isolates in the total percentage of infected BMMø and BMDC (Figure 1A-B). However, significant differences were observed in BMDC at $24 \mathrm{~h}$ and $72 \mathrm{~h}$ after infection (see Figure 1B). Nevertheless, the analysis
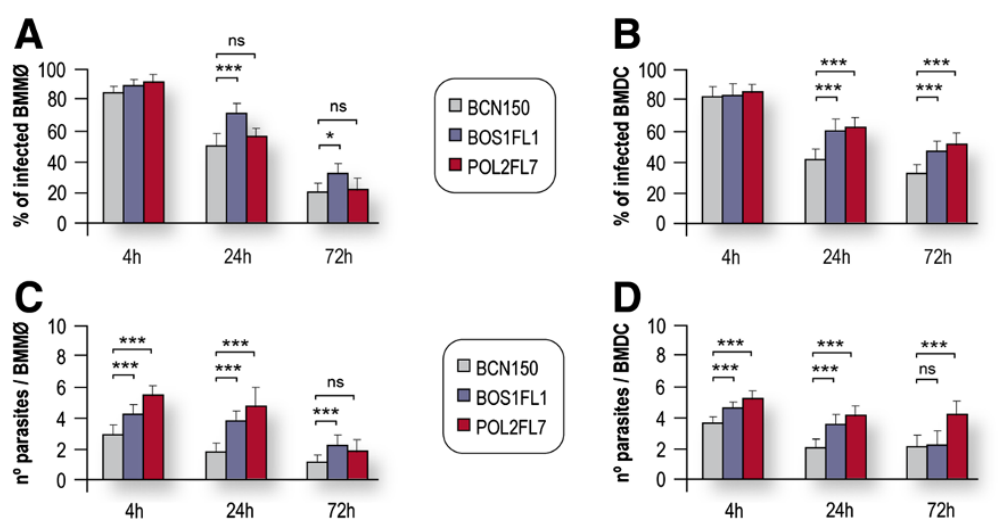

Figure 1 Ex vivo differential virulence of $\boldsymbol{L}$. infantum parasites. The time course of infection was followed counting (A, B) infected cells and (C, D) the number of parasites per infected cell. In cases in which the data was not normally distributed $(P<0.05)$, a 2-sample t-test could not be used. We used the Mann-Whitney $U$ test instead. Each value represents the mean \pm SD from three independent experiments. Significant differences between BCN150 strain and the other isolates were determined, as indicated. ${ }^{*} P<0.05,{ }^{* *} P<0.01,{ }^{* *} P<0.001$; ns, not significant. 
Table 1 Infection indeces

\begin{tabular}{|c|c|c|c|c|c|c|}
\hline \multirow[t]{2}{*}{ Infection index } & \multicolumn{3}{|l|}{ ВMМФ } & \multicolumn{3}{|l|}{ BMDC } \\
\hline & BCN150 & BOS1FL1 & POL2FL7 & BCN150 & BOS1FL1 & POL2FL7 \\
\hline $4 \mathrm{~h}$ after infection & $252 \pm 50$ & $396 \pm 50 *$ & $552 \pm 50 *$ & $319 \pm 25$ & $399 \pm 30^{*}$ & $475 \pm 50^{*}$ \\
\hline $24 \mathrm{~h}$ after infection & $110 \pm 50$ & $300 \pm 50^{*}$ & $275 \pm 50^{*}$ & $115 \pm 25$ & $247 \pm 20^{*}$ & $312 \pm 30^{*}$ \\
\hline $72 \mathrm{~h}$ after infection & $30 \pm 10$ & $60 \pm 15^{*}$ & $40 \pm 10^{*}$ & $102 \pm 10$ & $137 \pm 15^{*}$ & $292 \pm 20^{*}$ \\
\hline
\end{tabular}

In order to determine the overall parasite burdens, an infection index value was calculated for each time after infection by multiplying the mean percentage of infected cells by the mean number of amastigotes per infected cell. The resulting values are expressed in arbitrary units. One representative experiment out of three is shown. The mean \pm SD are shown. ${ }^{*} P<0.05$.

of parasite load per infected cell showed that both BOS1FL1 and POL2FL7 isolates were more effective in the invasion of BMMø and BMDC compared to the $\mathrm{BCN} 150$ strain. In this study, the median values of parasite load per BCN150-infected cells were considered representative samples (reference values) of our experiments using this well-characterized L. infantum strain. As expected at $24 \mathrm{~h}$ after infection [29,31], we detected a reduction in BCN150-infected the intracellular parasite load (for all three strains) compared to cells at the initial time point of infection (Figure 1C-D), which was more apparent in BMMø and BMDC. As previously described [31], the number of BMDC containing intracellular parasites dropped significantly between $4 \mathrm{~h}$ to $24 \mathrm{~h}$. After the slight decrease observed at $24 \mathrm{~h}$, the mean number of amastigotes per infected BMDC decreased at later time points, although the parasite burdens in cells infected with the BOS1FL1 and POL2FL7 isolates remained greater compared to cells infected with the BCN150 strain. Therefore, both the BOS1FL1 and POL2FL7 isolates were more resistant to BMMø and BMDC leishmanicidal activities, as displayed by significantly higher values of infection indices at all time points studied, compared to those observed in BCN150 infection (Table 1). Thus, differences in amastigotes content were clearly visible under a light microscope after $24 \mathrm{~h}$ of infection (Figure 2). Given these results,
BOS1FL1 and POL2FL7 isolates may be considered to be more infective than the BCN150 strain.

\section{Characterisation of the quality of immune response due to ex vivo infection}

Infection with $L$. infantum is known to lead to cytokine secretion by host cells that will dictate the nature of T-cell responses. The quality of these immune responses is influenced by the equilibrium between the production of pro-inflammatory cytokines (mainly represented by IL-12) and anti-inflammatory cytokines (mainly represented by IL-10). In VL, IL-10 suppresses IFN- $\gamma$ and IL-12 production, while disease regression is associated with optimal IL-12p40 production by DC and Mø that drives a predominant $\mathrm{T}$ helper 1 response. Th1 $\mathrm{T}$ cell responses result in IFN- $\gamma$ production, type 1 antibody responses, classical macrophage activation and subsequent NO-mediated killing effector functions [32,33]. These activities are essential to destroy the invading pathogens $[34,35]$.

Thus, controlled parallel experiments were performed in the ex vivo murine model of infection to assess the ability of parasites (BOS1FL1, POL2FL7 and BCN150) to modulate cytokine synthesis in LPS- activated BMMø and BMDC. Leishmania infection induced lower amounts of IL-12p40 production compared to amounts observed

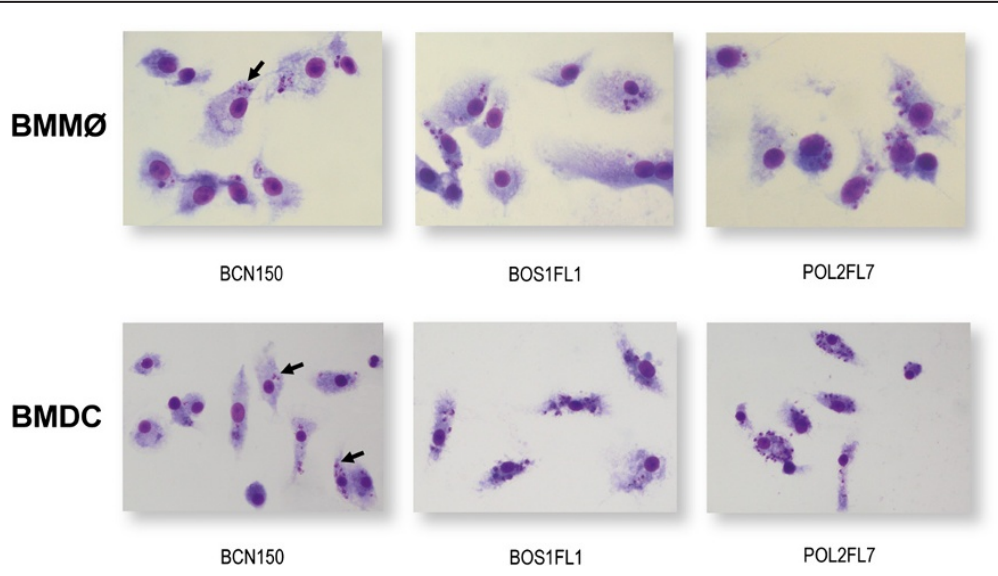

Figure 2 Examination of infection of BMMø and BMDC at $\mathbf{2 4} \mathrm{h}$ after infection. Infected cultures were washed to remove free parasites, fixed in methanol, stained with Giemsa and examined under a light microscope. Intracellular amastigotes (arrows) were clearly visible. 


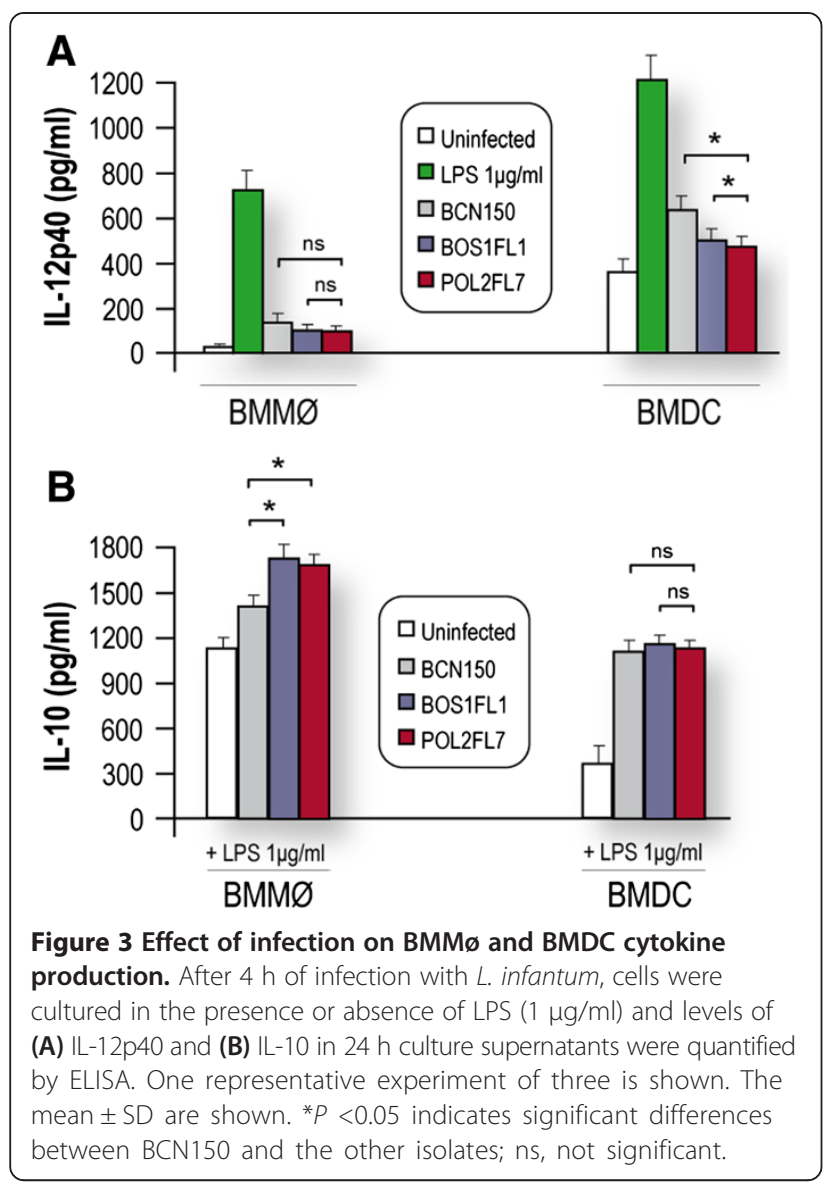

when cells were stimulated with only LPS (Figure 3A), which is consistent with previous research [36]. Interestingly, the production of IL-12p40 was decreased mainly in BOS1FL1 - and POL2FL7-infected BMMø and BMDC $(P<0.05)$, compared to amounts observed during infection with the BCN150 strain (Figure 3A). BMMø infected with BOS1FL1 and POL2FL7 isolates produced higher levels of IL-10 $(P<0.05)$ compared to those produced in cultures of cells infected with the BCN150 strain. In contrast, no significant differences were found for the IL-10 levels in BMDC after $24 \mathrm{~h}$ of infection with any of three L. infantum strains (Figure 3B). Altogether, these data show that BOS1FL1- and POL2FL7-infected BMMø produced higher levels of the anti-inflammatory cytokine IL-10, while these isolates interfered significantly in optimal IL-12p40 production by Leishmania-infected BMDC. These results suggest that there are two ways by which these isolates may alter the defences of host cells, depending on whether the infection involves BMMø or BMDC.

To investigate whether BOS1FL1, POL2FL7 or BCN150 parasites can affect host cell responses that kill intracellular parasites, we compared the levels of NO produced and arginase activity in ex vivo-infected BMMø and BMDC at $96 \mathrm{~h}$ after infection. We found significantly reduced NO production (Figure 4A) in supernatants from BOS1FL1- and POL2FL7-infected BMMø and BMDC in the presence of LPS compared to those obtained in BCN150-infected cells. The results also showed that ex vivo infections lead to augmented arginase activity in infected cells, and confirmed previous investigations using different Leishmania species [37-39]. In agreement with data above, arginase activity was increased in BMMø and BMDC infected with BOS1FL1 and POL2FL7 (Figure 4B), compared to levels of activity in cells infected with $\mathrm{BCN150}$. It is well known that arginase, an enzyme induced by Th2 cytokines, is a hallmark of host cells and is responsible not only for the transformation of L-arginine into polyamines, contributing to the growth of Leishmania parasites, but arginase is also important for parasite infectivity [40]. This increased arginase activity in phagocytic cells is believed to deplete L-arginine availability and to competitively inhibit iNOS activity and NO production $[37,41,42]$. Data in the present study clearly show that the increased arginase activity in BMMø and BMDC infected
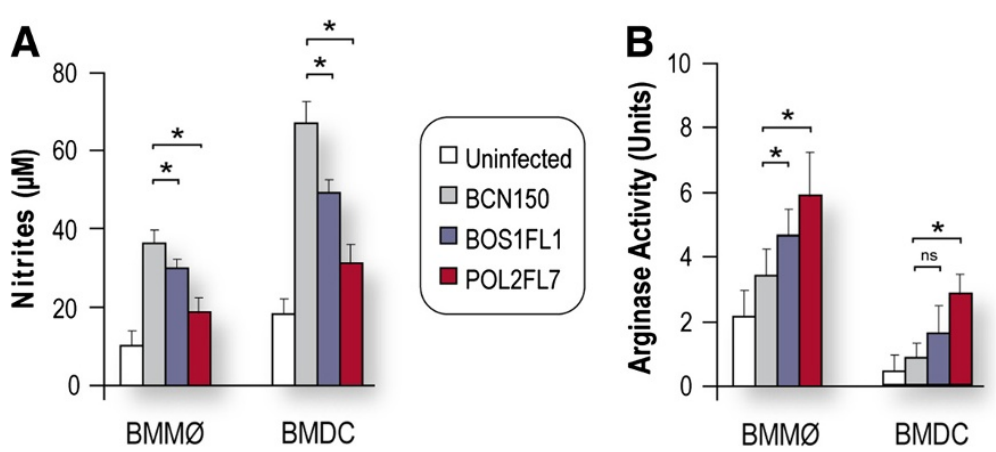

Figure $4 \mathrm{NO}$ release and arginase activity at $96 \mathrm{~h}$ after infection. (A) Immediately after $4 \mathrm{~h}$ of infection, cells were stimulated with LPS (1 $\mu \mathrm{g} / \mathrm{ml})$ and nitrite levels in culture supernatants harvested 96 hours later were determined using Griess assay method. (B) Non-stimulated cells were lysed and the total arginase activity was determined. Data are presented as mean \pm SD and are representative of three different experiments with similar results. ${ }^{*} P<0.05$ indicates significant differences between BCN150 and the other isolates; ns, not significant. 
with BOS1FL1 and POL2FL7 is likely to overcome the parallel NO release, resulting in reduced leishmanicidal activity in host cells.

\section{Conclusions}

This study was designed to examine the ex vivo virulence of L. infantum strains isolated from an area associated with human leishmaniosis in Madrid and to assess the potential implications in this human leishmaniosis outbreak. Our data indicate that $L$. infantum isolates from an endemic area exhibited high virulence in terms of infection index, cytokine production and enzymatic activities involved in the pathogenesis of VL. These data provide a starting point for the study of the virulence behaviour of parasite isolates (BOS1FL1 and POL2FL7) from an outbreak of human leishmaniosis in Madrid (Spain), including their involvement in infecting immunocompetent hosts. Further studies will be needed to determine whether these findings are observed in an in vivo immunocompetent murine model of VL.

\section{Competing interests}

The authors declare that they have no competing interests.

\section{Authors' contributions}

GDB help to design he study, participated in the in vitro studies and helped draft the manuscript. MJ and RM obtained parasite isolates from P. perniciosus captured in the area, participated in data collection and analyses, and helped to draft the manuscript. LOG performed image capture using optical microscopy and helped draft the manuscript. AMR and AM helped to carry out ex vivo infections and participated in the microscopic examination of samples. MTC helped to draft the manuscript. JC conceived and coordinated the study, participated in its design and in the in vitro assays, helped to perform the statistical analyses and drafted the manuscript. All authors read and approved the final manuscript.

\section{Acknowledgements}

This study was supported by Grants AGL2010-17394 and AGL2013-44100R from the Spanish Ministry of Economy and Competitiveness and was partially funded by EU grant FP7-2011-261504 EDENext and the paper is catalogued by the EDENext Steering Committee as EDENext 276 (http://www.edenext.eu).

\section{Author details \\ 'Department of Animal Health, Faculty of Veterinary Science, Complutense University of Madrid, 28040 Madrid, Spain. ${ }^{2}$ Medical Entomology Unit, Department of Parasitology, National Centre of Microbiology, Carlos III Institute of Health, Majadahonda 28220, Madrid, Spain. " "Severo Ochoa" Molecular Biology Centre CSIC-UAM, 28049 Madrid, Spain.}

Received: 2 August 2014 Accepted: 23 October 2014

Published online: 07 November 2014

\section{References}

1. Gouzelou E, Haralambous C, Antoniou M, Christodoulou V, Martinkovic F, Zivicnjak T, Smirlis D, Pratlong F, Dedet JP, Ozbel Y, Toz S, OPresber W, Schonian G, Soteriadou K: Genetic diversity and structure in Leishmania infantum populations from southeastern Europe revealed by microsatellite analysis. Parasit Vectors 2013, 6:342.

2. Gramiccia M: Recent advances in leishmaniosis in pet animals: epidemiology, diagnostics and anti-vectorial prophylaxis. Vet Parasitol 2011, 181(1):23-30.

3. Dujardin JC, Campino L, Canavate C, Dedet JP, Gradoni L, Soteriadou K, Mazeris A, Ozbel Y, Boelaert M: Spread of vector-borne diseases and neglect of Leishmaniasis. Eur Emerg Infect Dis 2008, 14(7):1013-1018.
4. Solano-Gallego L, Koutinas A, Miro G, Cardoso L, Pennisi MG, Ferrer L, Bourdeau P, Oliva G, Baneth G: Directions for the diagnosis, clinical staging, treatment and prevention of canine leishmaniosis. Vet Parasitol 2009, 165(1-2):1-18.

5. Maia C, Campino L: Can domestic cats be considered reservoir hosts of zoonotic leishmaniasis? Trends Parasitol 2011, 27(8):341-344.

6. Maia C, Ramos C, Coimbra M, Bastos F, Martins A, Pinto P, Nunes M, Vieira ML, Cardoso L, Campino L: Bacterial and protozoal agents of feline vector-borne diseases in domestic and stray cats from southern Portugal. Parasit Vectors 2014, 7:115.

7. Miro G, Ruperez C, Checa R, Galvez R, Hernandez L, Garcia M, Canorea I, Marino V, Montoya A: Current status of L. infantum infection in stray cats in the Madrid region (Spain): implications for the recent outbreak of human leishmaniosis? Parasit Vectors 2014, 7:112.

8. Efstathiou A, Gaboriaud-Kolar N, Smirlis D, Myrianthopoulos V, Vougogiannopoulou K, Alexandratos A, Kritsanida M, Mikros E, Soteriadou K, Skaltsounis AL: An inhibitor-driven study for enhancing the selectivity of indirubin derivatives towards leishmanial Glycogen Synthase Kinase-3 over leishmanial cdc2-related protein kinase 3. Parasit Vectors 2014, 7(1):234.

9. Kobets T, Grekov I, Lipoldova M: Leishmaniasis: prevention, parasite detection and treatment. Curr Med Chem 2012, 19(10):1443-1474.

10. Gradoni L, Soteriadou K, Louzir H, Dakkak A, Toz SO, Jaffe C, Dedet JP, Campino L, Canavate C, Dujardin JC: Drug regimens for visceral leishmaniasis in Mediterranean countries. Trop Med Int Health 2008, 13(10):1272-1276.

11. Gramiccia M, Gradoni L: The current status of zoonotic leishmaniases and approaches to disease control. Int J Parasitol 2005, 35(11-12):1169-1180.

12. Tilman D, Lehman C: Human-caused environmental change: impacts on plant diversity and evolution. Proc Natl Acad Sci U S A 2001, 98(10):5433-5440.

13. Aguado M, Espinosa P, Romero-Mate A, Tardio JC, Cordoba S, Borbujo J: Outbreak of Cutaneous Leishmaniasis in Fuenlabrada, Madrid. Actas Dermosifiliogr 2013, 104(4):334-342.

14. Jimenez M, Gonzalez E, Martin-Martin I, Hernandez S, Molina R: Could wild rabbits (Oryctolagus cuniculus) be reservoirs for Leishmania infantum in the focus of Madrid, Spain? Vet Parasitol 2014, 202(3-4):296-300.

15. Molina R, Jimenez MI, Cruz I, Iriso A, Martin-Martin I, Sevillano O, Melero S, Bernal J: The hare (Lepus granatensis) as potential sylvatic reservoir of Leishmania infantum in Spain. Vet Parasitol 2012, 190(1-2):268-271.

16. Moreno I, Alvarez J, Garcia N, de la Fuente S, Martinez I, Marino E, Toraño A, Goyache J, Vilas F, Dominguez L, Dominguez M: Detection of anti-Leishmania infantum antibodies in sylvatic lagomorphs from an epidemic area of Madrid using the indirect immunofluorescence antibody test. Vet Parasitol 2013, 199(3-4):264-267.

17. Garcia N, Moreno I, Alvarez J, de la Cruz ML, Navarro A, Perez-Sancho M, Garcia-Seco T, Rodriguez-Bertos A, Conty ML, Toraño A, Prieto A, Dominguez $L$, Dominguez M: Evidence of Leishmania infantum Infection in Rabbits (Oryctolagus cuniculus) in a Natural Area in Madrid. Spain Biomed Res Int 2014, 2014:318254.

18. Martin-Martin I, Molina R, Rohousova I, Drahota J, Volf P, Jimenez M: High levels of anti-Phlebotomus perniciosus saliva antibodies in different vertebrate hosts from the re-emerging leishmaniosis focus in Madrid, Spain. Vet Parasitol 2014, 202(3-4):207-216.

19. Jimenez M, Gonzalez E, Iriso A, Marco E, Alegret A, Fuster F, Molina R: Detection of Leishmania infantum and identification of blood meals in Phlebotomus perniciosus from a focus of human leishmaniasis in Madrid, Spain. Parasitol Res 2013, 112(7):2453-2459.

20. Chicharro C, Llanes-Acevedo IP, Garcia E, Nieto J, Moreno J, Cruz I: Molecular typing of Leishmania infantum isolates from a leishmaniasis outbreak in Madrid, Spain, 2009 to 2012. Euro Surveill 2013, 18(30):20545.

21. Fernandez-Cotrina J, Iniesta V, Belinchon-Lorenzo S, Muñoz-Madrid R, Serrano F, Parejo JC, Gomez-Gordo L, Soto M, Alonso C, Gomez-Nieto LC: Experimental model for reproduction of canine visceral leishmaniosis by Leishmania infantum. Vet Parasitol 2012, 192(1-3):118-128.

22. Carrion J, Nieto A, Iborra S, Iniesta V, Soto M, Folgueira C, Abanades DR, Requena JM, Alonso C: Immunohistological features of visceral leishmaniasis in BALB/c mice. Parasite Immunol 2006, 28(5):173-183.

23. Carrion J, Folgueira C, Alonso C: Immunization strategies against visceral leishmaniosis with the nucleosomal histones of Leishmania infantum encoded in DNA vaccine or pulsed in dendritic cells. Vaccine 2008, 26(20):2537-2544. 
24. Nieto A, Dominguez-Bernal G, Orden JA, De La Fuente R, Madrid-Elena N, Carrion J: Mechanisms of resistance and susceptibility to experimental visceral leishmaniosis: BALB/c mouse versus Syrian hamster model. Vet Res 2011, 42:39.

25. Molano I, Alonso MG, Miron C, Redondo E, Requena JM, Soto M, Nieto CG, Alonso C: A Leishmania infantum multi-component antigenic protein mixed with live BCG confers protection to dogs experimentally infected with L. infantum. Vet Immunol Immunopathol 2003, 92(1-2):1-13.

26. Carcelen J, Iniesta V, Fernandez-Cotrina J, Serrano F, Parejo JC, Corraliza I, Gallardo-Soler A, Marañon F, Soto M, Alonso C, Gomez-Nieto C: The chimerical multi-component $\mathrm{Q}$ protein from Leishmania in the absence of adjuvant protects dogs against an experimental Leishmania infantum infection. Vaccine 2009, 27(43):5964-5973.

27. Marim FM, Silveira TN, Lima DS Jr, Zamboni DS: A method for generation of bone marrow-derived macrophages from cryopreserved mouse bone marrow cells. PLoS One 2010, 5(12):e15263.

28. Carrion J, Nieto A, Soto M, Alonso C: Adoptive transfer of dendritic cells pulsed with Leishmania infantum nucleosomal histones confers protection against cutaneous leishmaniosis in BALB/c mice. Microbes Infect 2007, 9(6):735-743.

29. Cunha J, Carrillo E, Sanchez C, Cruz I, Moreno J, Cordeiro-da-Silva A: Characterization of the biology and infectivity of Leishmania infantum viscerotropic and dermotropic strains isolated from HIV + and HIV- patients in the murine model of visceral leishmaniasis. Parasit Vectors 2013, 6:122

30. Corraliza IM, Campo ML, Soler G, Modolell M: Determination of arginase activity in macrophages: a micromethod. J Immunol Methods 1994, 174(1-2):231-235.

31. Neves BM, Silvestre R, Resende M, Ouaissi A, Cunha J, Tavares J, Loureiro I, Santarem N, Silva AM, Lopes MC, Cruz MT, Cordeiro Da Silva A: Activation of phosphatidylinositol 3-kinase/Akt and impairment of nuclear factor-kappaB: molecular mechanisms behind the arrested maturation/ activation state of Leishmania infantum-infected dendritic cells. Am J Pathol 2010, 177(6):2898-2911.

32. Vieira LQ, Hondowicz BD, Afonso LC, Wysocka M, Trinchieri G, Scott P: Infection with Leishmania major induces interleukin-12 production in vivo. Immunol Lett 1994, 40(2):157-161.

33. Bacellar O, D'Oliveira A Jr, Jeronimo S, Carvalho EM: IL-10 and IL-12 are the main regulatory cytokines in visceral leishmaniasis. Cytokine 2000, 12(8):1228-1231.

34. Brombacher F, Kastelein RA, Alber G: Novel IL-12 family members shed light on the orchestration of Th1 responses. Trends Immunol 2003, 24(4):207-212.

35. Guler R, Afshar M, Arendse B, Parihar SP, Revaz-Breton M, Leitges M, Schwegmann A, Brombacher F: PKCdelta regulates IL-12p40/p70 production by macrophages and dendritic cells, driving a type 1 healer phenotype in cutaneous leishmaniasis. Eur J Immunol 2011, 41(3):706-715.

36. Resende M, Moreira D, Augusto J, Cunha J, Neves B, Cruz MT, Estaquier J, Cordeiro-da-Silva A, Silvestre R: Leishmania-infected MHC class Ilhigh dendritic cells polarize CD4+ T cells toward a nonprotective T-bet + IFN-gamma + IL-10+ phenotype. J Immunol 2013, 191(1):262-273.

37. Iniesta V, Gomez-Nieto LC, Corraliza I: The inhibition of arginase by $\mathrm{N}$ (omega)-hydroxy-l-arginine controls the growth of Leishmania inside macrophages. J Exp Med 2001, 193(6):777-784.

38. Muleme HM, Reguera RM, Berard A, Azinwi R, Jia P, Okwor IB, Beverley S, Uzonna JE: Infection with arginase-deficient Leishmania major reveals a parasite number-dependent and cytokine-independent regulation of host cellular arginase activity and disease pathogenesis. J Immunol 2009, 183(12):8068-8076.

39. Ehrchen J, Helming L, Varga G, Pasche B, Loser K, Gunzer M, Sunderkotter C, Sorg C, Roth J, Lengeling A: Vitamin D receptor signaling contributes to susceptibility to infection with Leishmania major. FASEB J 2007, 21(12):3208-3218.

40. da Silva MF, Zampieri RA, Muxel SM, Beverley SM, Floeter-Winter LM: Leishmania amazonensis arginase compartmentalization in the glycosome is important for parasite infectivity. PLOS One 2012, 7(3):e34022.

41. Kropf P, Fuentes JM, Fahnrich E, Arpa L, Herath S, Weber V, Soler G, Celada A, Modolell M, Muller l: Arginase and polyamine synthesis are key factors in the regulation of experimental leishmaniasis in vivo. FASEB J 2005, 19(8):1000-1002.

42. Iniesta V, Gomez-Nieto LC, Molano I, Mohedano A, Carcelen J, Miron C, Alonso C, Corraliza I: Arginase I induction in macrophages, triggered by Th2-type cytokines, supports the growth of intracellular Leishmania parasites. Parasite Immunol 2002, 24(3):113-118.

doi:10.1186/s13071-014-0499-1

Cite this article as: Domínguez-Bernal et al:: Characterisation of the ex vivo virulence of Leishmania infantum isolates from Phlebotomus perniciosus from an outbreak of human leishmaniosis in Madrid, Spain. Parasites \& Vectors 2014 7:499.

\section{Submit your next manuscript to BioMed Central and take full advantage of:}

- Convenient online submission

- Thorough peer review

- No space constraints or color figure charges

- Immediate publication on acceptance

- Inclusion in PubMed, CAS, Scopus and Google Scholar

- Research which is freely available for redistribution

Submit your manuscript at www.biomedcentral.com/submit
C Biomed Central 